\title{
News Headline Grouping as a Challenging NLU Task
}

\author{
Philippe Laban \\ UC Berkeley
}

\author{
Lucas Bandarkar \\ UC Berkeley
}

\author{
Marti A. Hearst* \\ UC Berkeley
}

\begin{abstract}
Recent progress in Natural Language Understanding (NLU) has seen the latest models outperform human performance on many standard tasks. These impressive results have led the community to introspect on dataset limitations, and iterate on more nuanced challenges. In this paper, we introduce the task of HeadLine Grouping (HLG) and a corresponding dataset (HLGD) consisting of 20,056 pairs of news headlines, each labeled with a binary judgement as to whether the pair belongs within the same group. On HLGD, human annotators achieve high performance of around 0.9 F-1, while current state-of-the art Transformer models only reach $0.75 \mathrm{~F}-1$, opening the path for further improvements. We further propose a novel unsupervised Headline Generator Swap model for the task of HeadLine Grouping that achieves within 3 F-1 of the best supervised model. Finally, we analyze highperforming models with consistency tests, and find that models are not consistent in their predictions, revealing modeling limits of current architectures.
\end{abstract}

\section{Introduction}

Headlines are a key component in everyday news consumption. As the first piece of text the user interacts with when learning about a story, the headline can play many roles, including: summarize the main points of the story, promote a particular detail, and convince the reader to choose one source over another (Bonyadi and Samuel, 2013).

News aggregators amass content from many disparate news sources and have become popular, in part because they offer news readers access to diverse sources (Chowdhury and Landoni, 2006). Flaxman et al. (2016) find that news aggregators help news readers access content they are unfamiliar with, and potentially on opposite sides of the \footnotetext{
* Author emails: \{phillab, lucasban-
} darkar,hearst\}@berkeley.edu

\begin{tabular}{|c|c|c|}
\hline Date & Headline & Group \\
\hline $03 / 276$ & $\begin{array}{l}\text { Russian-U.S. crew makes belated } \\
\text { arrival at space station }\end{array}$ & A \\
\hline $03 / 28 \sqrt{240 ;}$ & $\begin{array}{l}\text { Russian spacecraft brings 3-man } \\
\text { crew to ISS after } 2 \text {-day delay }\end{array}$ & A \\
\hline 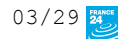 & Space 'makes the heart grow rounder' & B \\
\hline
\end{tabular}

Figure 1: Snippet of timeline in the HeadLine Grouping dataset (HLGD). The headlines are part of the Space timeline, one of 10 timelines in HLGD. Headlines labeled A are part of a group; those labeled B are part of another group.

political spectrum. At the heart of a news aggregator is the ability to group relevant content together, to support a reader in finding varying views and angles on the news.

Natural Language Understanding (NLU) has seen rapid progress in recent years. The creation of multi-task benchmarks such as the General Language Understanding Evaluation collection (GLUE), paired with fast-paced progress in Transformer-based architectures has led to models outperforming human baseline performance on many tasks, such as paraphrase identification (Dolan et al., 2004), semantic similarity (Cer et al., 2017), and extractive question-answering (QA) (Rajpurkar et al., 2018).

This success has led to the questioning of the composition of benchmarks, and the subsequent creation of ever-more challenging datasets, for example by increasing the diversity of texts in textual entailment datasets (Williams et al., 2018), or introducing unanswerable questions in QA datasets (Rajpurkar et al., 2018).

\subsection{HeadLine Grouping Definition}

In this paper, we propose the novel task of HeadLine Grouping. Although news articles may discuss several topics, because of length constraints, headlines predominantly describe a single event. 
Therefore, for the task of headline grouping, we define two headlines to be in the same group if they describe the same event: an action that occurred at a specific time and place. We do not require headlines to contain fully identical information to be placed into the same group. For example, one headline might report an exact number of deaths, while another might report a rounded number, or omit the number altogether. Figure 1 shows an example from our dataset. The first two headlines are in group A, and the third and fourth are part of group $\mathrm{B}$. The headlines are divided into groups A versus B because they describe different events in the timeline (astronauts arriving at the space station vs. a study about hearts in space). The two headlines in B show the lexical and syntactic diversity of groups in this dataset - they appear in the same group because they describe the same underlying event. Appendix D gives a longer excerpt.

We build a large dataset for the task of HeadLine Grouping, crowd-sourcing the annotation of large timelines of news headlines in English. We cast the task as a binary classification: given a pair of headlines, determine whether they are part of a headline group (1) or whether they relate to distinct events (0).

\subsection{Contributions}

Our main contribution, described in Section 3, is the design of the HeadLine Grouping task (HLG), and the creation of the HeadLine Grouping Dataset (HLGD) that is focused on detecting when headlines refer to the same underlying event. We show that the human annotations in our dataset have strong inter-annotator agreement (average 0.81), and a human annotator can achieve high performance on our corpus (around 0.9 F-1), while current state-of-the-art Transformer-based model performance stands around $0.75 \mathrm{~F}-1$.

A second contribution is a novel unsupervised approach for the task of HeadLine Grouping relying on modifying a headline generator model. The model achieves the best performance on HLGD amongst unsupervised methods. Section 4 presents the performance of this algorithm compared to several baselines, including supervised and unsupervised methods.

Our final contribution, presented in Section 5, is an analysis of the consistency of the best performing model on HLGD. We specifically analyze whether the model follows commutative and transi- tive behavior expected to be trivially true in HeadLine Grouping. ${ }^{1}$

\section{Related Work}

Paraphrase Identification, Textual Entailment and Semantic Similarity are three common NLP tasks that resemble HeadLine Grouping. In Paraphrase Identification (PI) (Ozmutlu, 2016; Xu et al., 2014), the objective is to determine whether two sentences are semantically equivalent. We show in Table 2 that only one third of positive headline pairs in HLGD qualify as paraphrases. We further show in Section 4 that a trained model on MRPC (Dolan et al., 2004), a PI dataset of news text, performs poorly on HLGD. Textual entailment (Bentivogli et al., 2009), or Natural Language Inference (NLI) (Williams et al., 2018), determines whether a premise text implies a hypothesis. Apart from the non-symmetricality of the entailment relationship, we believe entailment is not well-suited to the domain of headlines because of the strict nature of the relationship. A large portion of headlines in a group differ in level of detail, and under an entailment task, would need to be labeled as neutral or contradicting. Finally, semantic similarity assigns a strength of similarity between two candidate sentences, for example in the Semantic Textual Similarity Benchmark (STS-B) (Cer et al., 2017), similarity is ranked from 1 to 5. This flexibility seems like a good fit; however, the lexical and syntactic diversity of headlines about the same underlying content do not correspond well to a similarity range.

Topic Detection and Tracking (TDT) (Allan, 2002) was a DARPA-sponsored initiative to investigate methods to group news articles by topics (referred to as timelines in this paper). We view TDT as a precursor to the task of HeadLine Grouping: in TDT, the focus is on detecting and tracking a timeline of related events, while in HeadLine Grouping, the timeline is given, and the focus is on subdividing it into finer groups. We considered using the TDT datasets and annotating them for our purposes. However, the TDT developers acknowledge (Graff et al., 2006) several important errors in the way the TDT datasets were acquired (e.g., some publication dates were not properly attributed) that could have an impact on the quality

\footnotetext{
${ }^{1}$ The code, model checkpoints and dataset are available at: https://github.com/tingofurro/headline_ grouping
} 
of the final dataset.

News Headlines in NLP. Headlines are popular as a challenging source for generation tasks such as summarization (Rush et al., 2015), style transfer (Jin et al., 2020), and style-preserving translation (Joshi et al., 2013). Headlines have been leveraged to detect political bias (Gangula et al., 2019), clickbait and fake news phenomena (Bourgonje et al., 2017). Finally, sentiment analysis of headlines has received attention (Bostan et al., 2020), with some work showing headline sentiment can be a useful signal in finance (Moore and Rayson, 2017).

Grouping Headlines has been explored in prior work. Wubben et al. (2009) propose a TF-IDF based clustering algorithm, but do not evaluate its agreement with human annotations. Pronoza et al. (2015) build a corpus of Russian headlines pairs, but limit pairs in the dataset by filtering out headlines that are distant syntactically. We find that headline groups often contain syntactically distant headlines (see Figure 3). Bouamor et al. (2012) and Shinyama et al. (2002) present a simple strategy, relying on the assumption that all articles on a topic published on the same day form a group. As will be shown below, this assumption is not always correct (see Figure 2).

Several of the most-used news aggregators, such as Yahoo News ${ }^{2}$, Google News ${ }^{3}$, and Bloomberg's NSTM (Bambrick et al., 2020) present headlines in groups. As these systems do not have published algorithms, we cannot comment on their methods; nonetheless we hope that the release of the HLG dataset offers a common evaluation test-bed to benchmark systems.

\section{HeadLine Grouping Dataset}

We now present the HeadLine Grouping Dataset. We describe the dataset of news articles we collected for annotation, our annotation procedure, an analysis of the resulting dataset, and the challenges we propose to the community.

\subsection{Dataset Source}

We collect a set of 10 news timelines from an existing open-source news collection in English (Laban and Hearst, 2017). A timeline is a collection of news articles about an evolving topic, consisting of a series of events. The timelines we use to build HLGD consist of time-stamped English news arti-

\footnotetext{
${ }^{2}$ https://news.yahoo.com

${ }^{3}$ https://news.google.com
}

\begin{tabular}{lcccl}
\hline Story Name & Size & Groups & + pairs & IAA \\
\hline Tunisia Protests & 111 & 46 & 219 & 0.758 \\
Ireland Abortion Vote & 180 & 81 & 406 & 0.727 \\
Ivory Coast Army Mutiny & 128 & 45 & 329 & 0.781 \\
International Space Station & 257 & 107 & 499 & 0.831 \\
US Bird Flu Outbreak & 79 & 36 & 91 & 0.924 \\
Human Cloning & 119 & 55 & 259 & 0.830 \\
\hline Facebook Privacy Scandal & 194 & 105 & 274 & 0.753 \\
Equifax Breach & 159 & 81 & 261 & 0.855 \\
\hline Brazil Dam Disaster & 273 & 132 & 634 & 0.818 \\
Wikileaks Trials & 180 & 101 & 550 & 0.859 \\
\hline Total / Average & $\mathbf{1 6 7 9}$ & $\mathbf{7 8 9}$ & $\mathbf{3 5 2 2}$ & $\mathbf{0 . 8 1 4}$ \\
\hline
\end{tabular}

Table 1: Names and statistics of the ten news timelines in HLGD. Size is the number of headlines in the timeline, Groups the number of distinct headline groups, + pairs the number of pairs of headlines in all groups, and IAA the inter-annotator agreement. Timelines are separated into training (1-6), development (78), and test (9-10).

cles originating from 34 international news sources. The timelines range in size from 80 to 274 news articles, and span 18 days to 10 years.

We choose to use timelines as the source for the dataset for two reasons. First, news timelines center around a theme, and as successive events occur, many pairs of headlines will be semantically close, yielding challenging samples for the dataset. Second, this task requires annotating headlines by pairs. If there are $n$ headlines, there could be on the order of $n^{2}$ headline pairs to annotate. By having annotators assign group labels to a chronologically organized timeline, the annotation procedure requires only one label per headline, or $n$ labels total.

We attempted to diversify topics and geographical locations represented in the 10 selected timelines. Topics and statistics are shown in Table 1.

\subsection{Annotation Procedure}

To reduce the effects of varying judgement inherent to the task, annotations were obtained from five independent judges and merged using the procedure described in the following subsection. Annotators worked on an entire timeline at a time, using the following procedure:

- The timeline was presented in a spreadsheet, in chronological order, with a single headline per row, and the corresponding publication date (year, month, day),

- Annotators went over the timeline one headline at a time in chronological order,

- If the headline being annotated did not match a previously created group, the annotator as- 
signed it a new group number,

- Otherwise, the annotator could assign the headline to a previous group number, grouping it with previously added headlines.

We note that the annotation relied on annotators' ability to discern an event described by a news headline. However, a headline is not always written in an event-centric manner - for example when the headline is vague (e.g., A way forward in gene editing in the Human Cloning timeline), or overly brief (e.g., Waste not, want not in the International Space Station timeline). Annotators were instructed to create a separate group for such cases, isolating non-event-centric headlines.

Roughly one fifth of the annotations were produced by authors of the paper, and the remaining annotations were obtained by recruiting 8 crowdworkers on the Upwork platform. ${ }^{4}$ The crowdworkers were all native English speakers with experience in either proof-reading or data-entry, and were remunerated at $\$ 14 /$ hour.

Annotators were first trained by reading a previously annotated timeline, and given the opportunity to clarify the task before starting to annotate. Exact instructions given to the annotators are transcribed in Appendix A.

\subsection{Merging Annotations}

In order to merge the five annotations, we follow a standard procedure to produce a single grouping that represents an aggregate of annotations.

We create a graph $G$, with each headline in a timeline represented by a node $n_{i}$. An edge $\left(n_{i}, n_{j}\right)$ is added to $G$ if a majority of the annotators (three or more of the five) put the two headlines in the same group. We apply a community detection algorithm, the Louvain method (Blondel et al., 2008), to $G$ to obtain a grouping of the headlines that we call the global groups.

\subsection{Inter-annotator Agreement}

We compare the groups of each annotator to the global groups for each timeline, and measure agreement between annotator groups and a leave-one-out version of the global groups using the standard Adjusted Mutual Information (Vinh et al., 2010). The average inter-annotator agreement is 0.814 , confirming that consensus amongst annotators is high. Inter-annotator agreement is reported for each timeline in Table 1.

\footnotetext{
${ }^{4}$ https://www.upwork.com
}

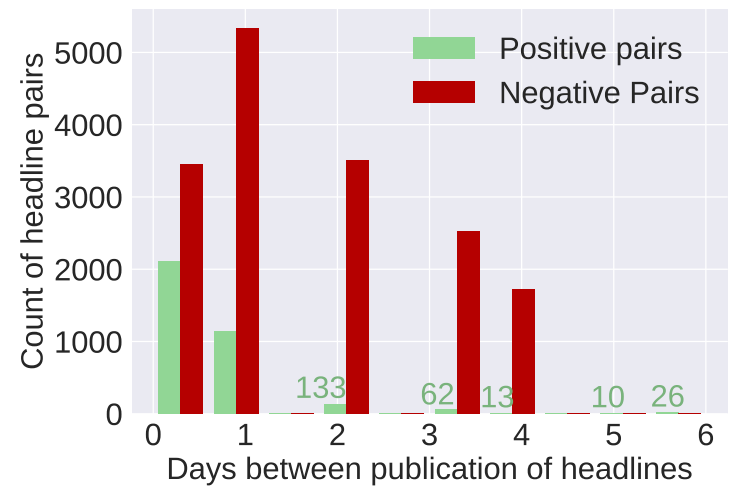

Figure 2: Histogram of difference in publication dates of positive and negative pairs in final dataset. Most positive headline pairs are published on the same day or within one day of each other. We down-sample negative pairs to only keep headlines published within 4 days of each other.

Section 4 provides individual annotator performance on HLGD, which obtain the highest performance of about $0.9 \mathrm{~F}-1$, further confirming that the task is well defined for human annotators.

\subsection{Creating the Final Dataset}

We transform the global groups into a binary classification task by generating pairs of headlines in the timelines: labeling the pair with a 1 if it belongs to the same group, and 0 otherwise.

With this procedure, we obtain 3,522 distinct positive headline pairs, and 154,156 negative pairs. This class imbalance is expected: two headlines picked at random in a timeline are unlikely to be in the same group. In order to reduce class imbalance, we down-sample negative pairs in the dataset.

Figure 2 shows the distribution of differences in publication dates for pairs of headlines in the final dataset. Publication date is indeed a strong signal to determine whether headlines are in the same group, as most positive pairs are published on the same day or one day apart. However, we show in Section 4 that using time as a sole indicator is not enough to perform well on the dataset.

In Figure 2, it can also be observed that $98 \%$ of positive headline pairs are published within 4 days of each other. Therefore, we only retain negative pairs that are within a 4 day window, filtering out simpler negative pairs from the final dataset. This final dataset has a class imbalance of roughly 1 positive pair to 5 negative pairs, for a total of 20,056 of labeled headlines pairs. This is similar in size to other NLU datasets, such as MRPC $(5,801$ 


\begin{tabular}{|c|c|c|c|}
\hline \multicolumn{4}{|c|}{ Positive Examples } \\
\hline Reasoning & Description & Example Headline Pair & Percentage \\
\hline $\begin{array}{l}\text { Difference in } \\
\text { Detail }\end{array}$ & $\begin{array}{l}\text { A headline conveys additional de- } \\
\text { tails, such as a name, or a cause }\end{array}$ & $\begin{array}{l}\text { NASA delays work on Moon rocket during virus pandemic } \\
\text { Nasa's Moon plans take a hit }\end{array}$ & $37 \%$ \\
\hline $\begin{array}{l}\text { Exact Para- } \\
\text { phrase }\end{array}$ & $\begin{array}{l}\text { Both headlines convey the same } \\
\text { information }\end{array}$ & $\begin{array}{l}\text { Equifax takes web page offline after reports of new cyber attack } \\
\text { Equifax takes down web page after reports of new hack }\end{array}$ & $30 \%$ \\
\hline $\begin{array}{l}\text { Difference in } \\
\text { Focus }\end{array}$ & $\begin{array}{l}\text { Headlines focus on a different as- } \\
\text { pect of the event group }\end{array}$ & $\begin{array}{l}\text { Astronauts to Get Thanksgiving Feast in Space } \\
\text { A Brief History of Thanksgiving Turkey in Space }\end{array}$ & $26 \%$ \\
\hline $\begin{array}{l}\text { Pun, Play-on- } \\
\text { word, etc. }\end{array}$ & $\begin{array}{l}\text { A headline has a unique stylistic } \\
\text { element to attract readers }\end{array}$ & $\begin{array}{l}\text { New privacy law forces some U.S. media offline in Europe } \\
\text { US websites blacked out in Europe on 'Happy GDPR Day' }\end{array}$ & $7 \%$ \\
\hline \multicolumn{4}{|c|}{ "Negative Examples } \\
\hline Reasoning & Description & Example Headline Pair & Percentage \\
\hline $\begin{array}{l}\text { Independent } \\
\text { events }\end{array}$ & $\begin{array}{l}\text { Headlines describe two distinct } \\
\text { events involving common actors }\end{array}$ & $\begin{array}{l}\text { Brazil dam disaster leaves } 34 \text { dead, hundreds missing } \\
\text { Alert raised over imminent risk to another Brazil mining dam }\end{array}$ & $44 \%$ \\
\hline $\begin{array}{l}\text { Related Sub- } \\
\text { events }\end{array}$ & $\begin{array}{l}\text { Describing two events that are re- } \\
\text { lated, e.g., follow each other }\end{array}$ & $\begin{array}{l}\text { Irish abortion referendum voting opens } \\
\text { Ireland set to end abortion ban as exit polls signal landslide vote }\end{array}$ & $36 \%$ \\
\hline $\begin{array}{l}\text { Headline too } \\
\text { broad }\end{array}$ & $\begin{array}{l}\text { One of the headlines is too broad } \\
\text { to assign to a particular event }\end{array}$ & $\begin{array}{l}\text { Obama commutes Chelsea Manning sentence } \\
\text { Who is Chelsea Manning? - video profile }\end{array}$ & $16 \%$ \\
\hline $\begin{array}{l}\text { Borderline / } \\
\text { Noise }\end{array}$ & $\begin{array}{l}\text { Could be positive or negative, } \\
\text { based on interpretation }\end{array}$ & & $4 \%$ \\
\hline
\end{tabular}

Table 2: Results of a manual typology of a subset of HLGD. An analysis of 200 positive, and 200 negative same-day headline pairs reveals there are several reasons why headlines get grouped or not in our dataset.

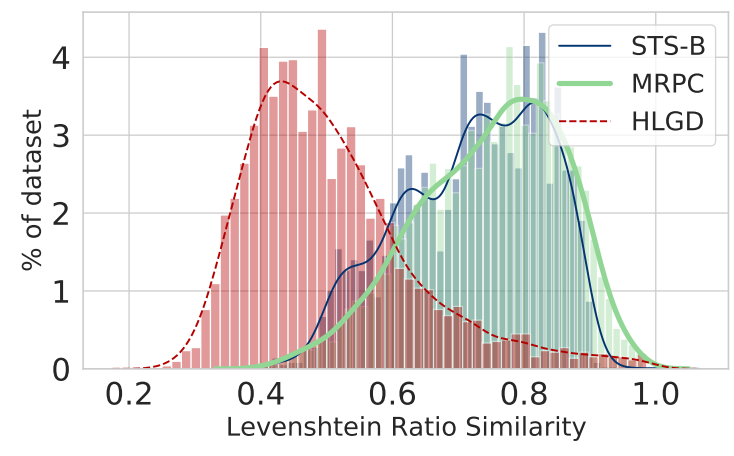

Figure 3: Levenshtein Similarity Distribution for positive pairs in MRPC, SST-B and HLGD. Pairs of sentences in HLGD can be positive examples (i.e., in the same group) while being less syntactically similar than in other NLU datasets such as STS-B or MRPC.

samples), or STS-B (8,628 samples).

Figure 3 shows the distribution of Levenshtein Ratio (Levenshtein, 1966) defined as:

$$
\operatorname{Ratio}\left(S_{1}, S_{2}\right)=1-\frac{\text { Levenshtein }\left(S_{1}, S_{2}\right)}{\max \left(\left|S_{1}\right|,\left|S_{2}\right|\right)}
$$

for positive pairs $\left(S_{1}, S_{2}\right)$ in MRPC and STS-B, two common NLU datasets, as well as HLGD, computed at the character level. The average similarity in HLGD (0.51) is lower than in the two others (0.72 and 0.74 , respectively). Furthermore, a classifier using solely the Levenshtein Ratio obtains an F-1 score of 0.81 on MRPC, but only 0.485 on HLGD. This suggests lexical distance alone does not contain a strong signal for good performance on HLGD.

\subsection{Analysis}

To gain insight into the linguistic phenomena that occur within and outside headline groups, the first author manually inspected 200 positive and 200 negative headline pairs in HLGD. Positive pairs were selected from randomly sampled large groups, and negative samples from same-day negative pairs, because headlines that appear on the same day but are not in the same group cannot be distinguished using time information and are likely to overlap semantically the most. In Table 2 , we list the phenomena we observed, give an example for each, and show the frequency in our sample. Within a group, headlines can be exact paraphrases, differ in detail level, differ in the element of focus, or involve stylistic elements such as puns. Negative headline pairs analyzed were either about independent events, related sub-events or involved a headline that was not specific enough. Additionally, around $4 \%$ of the negative samples analyzed were judged as borderline, interpretable as either positive or negative, showing that some ambiguity in the task is unavoidable. We believe this diversity in phenomena are ingredients that make HeadLine Grouping challenging and interesting for NLU research. 


\subsection{Challenges}

To allow for diversity in approaches to HeadLine Grouping, we propose to sub-divide HLGD into several challenges, limiting in each the data used to solve the classification task:

- Challenge 1: Headline-only. Access to the headline pairs only; similar to Paraphrase Identification and Textual Similarity tasks.

- Challenge 2: Headline + Time. Access to the headline pairs and their publication dates.

- Challenge 3: Headline + Time + Other. Access to the headline pairs, publication dates, and other information such as full content, author(s), and news source (a URL to the original article provides this access).

We believe these different challenges provide flexibility to probe a diversity of methods on the HLGD task. Challenge 1 fits the standard text-pair classification of NLU, similar to paraphrase identification, textual similarity and NLI, while additional meta-data available in Challenge 3 might be more compatible with the goals of the information retrieval community.

\section{Results on the Challenges}

In Table 3, we report the performance of a human annotator and a baseline, as well as unsupervised and supervised methods on HLGD. We chose Electra (Clark et al., 2020) for experiments based on a bi-directional Transformer (Vaswani et al., 2017), as initial experiments with other BERT (Devlin et al., 2019) variants performed similarly. Implementation details, model sizes and hyperparameters are listed in Appendix B.

\subsection{Human Performance and Baseline}

Human Performance reports the F-1 score of human annotators performing the task. Human performance is estimated by obtaining a sixth set of annotations for each timeline in the development and testing set, beyond the five used for dataset creation. These annotations were completed after several hours of practice on the training set timelines.

Human performance is distinct from the interannotator agreement (IAA) analysis presented in $\S 3.4$. IAA was performed on the five annotations used to create the dataset. We note that human

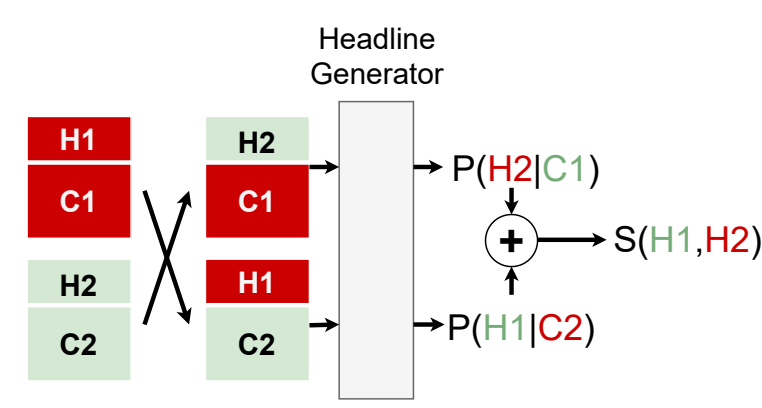

Figure 4: Schematic of the Headline Generator Swap model. We adapt a headline generator - a finetuned GPT-2 - to the task of HeadLine Grouping. The score of a pair of headlines is based on whether the generative model believes a swap of headlines is likely.

performance can theoretically achieve a perfect F1 score of 1.0 if the sixth annotator grouped the headlines identically to the global group.

Time only reports the performance of a logistic regression baseline based on the difference in days of publication between the two headlines. Data plotted in Figure 2 shows that a majority of positive pairs are published within two days of each other.

\subsection{Unsupervised Models}

Electra MRPC Zero-shot stands for an Electra model trained on the Microsoft Paraphrase Corpus (MRPC), achieving an F-1 of 0.92 on its development set. The objective is to evaluate whether a competitive paraphrase identification system achieves high performance on HLGD. The threshold to predict a label of one is tuned on the training portion of HLGD. This model only accesses headlines, and falls under Challenge 1 .

Electra MRPC Zero-shot + Time corresponds to the previous model, adding publication time into the model in the following way:

$$
P^{\prime}(Y=1 \mid X)=P(Y=1 \mid X) \cdot e^{-\lambda \Delta T}
$$

where $X$ represents the pair of headline inputs, $P(Y=1 \mid X)$ represents the model's confidence of the headline pair being in the same group, and $\Delta T$ the difference in days of publication of the headlines. $\lambda$ is tuned on the training set. Because this method leverages headline and time information, it falls under Challenge 2.

Headline Generator Swap is a novel approach we propose for zero-shot headline grouping, summarized in Figure 4.

Transformer-based Language Models like GPT2 (Radford et al., 2019) model the probability of 


\begin{tabular}{lccc}
\hline Method & Challenge & HLGD Dev F-1 & HLGD Test F-1 \\
\hline \multicolumn{4}{c}{ Human Performance and Baseline } \\
\hline Human Performance & 3 & $\mathbf{0 . 8 8 4}$ & $\mathbf{0 . 9 0 0}$ \\
Time-only & 2 & 0.654 & 0.585 \\
\hline \multicolumn{4}{c}{ Unsupervised / Zero-shot Models } \\
\hline Electra MRPC Zero-Shot & 1 & 0.562 & 0.626 \\
Electra MRPC Zero-Shot + Time & 2 & 0.666 & 0.688 \\
Headline Generator Swap & 3 & 0.671 & 0.651 \\
Headline Gen. Swap + Time & 3 & $\mathbf{0 . 7 2 7}$ & $\mathbf{0 . 7 2 2}$ \\
\hline \multicolumn{5}{c}{} \\
\hline Electra Finetune on HLGD & Supervised Models \\
Electra Finetune on HLGD content & 1 & 0.728 \\
Electra Finetune on HLGD + Time & 2 & 0.652 & 0.796 \\
\hline
\end{tabular}

Table 3: F-1 performance of several methods on the development and test portions of the HeadLine Grouping Dataset. Methods are separated into baselines: (1) the performance of a human annotator, and performance using only publication date, (2) unsupervised or zero-shot methods that do not leverage the training set for predictions, and (3) supervised methods. Each method falls under a challenge setting (1, 2 or 3$)$ based on data used.

a word sequence. As the first step in Headline Generator Swap, we use the GPT-2 model to create a headline generator to estimate the likelihood of a (headline, content) pair: $P_{L M}(H \mid C)$.

In more detail, we finetune a GPT- 2 model to read through the first 512 words of a news article and generate its headline. The headline generator is trained with teacher-forcing supervision, and a large corpus of 6 million (content, headline) pairs (Laban and Hearst, 2017), not overlapping HLGD.

The second step in Headline Generator swap is to use this probability to produce a symmetric score for two articles $A_{1}=\left(H_{1}, C_{1}\right)$ and $A_{2}=$ $\left(H_{2}, C_{2}\right)$ :

$$
S\left(A_{1}, A_{2}\right)=P_{L M}\left(H_{2} \mid C_{1}\right)+P_{L M}\left(H_{1} \mid C_{2}\right)
$$

This score evaluates the likelihood of a swap of headlines between articles $A_{1}$ and $A_{2}$, according to the GPT-2 language model. We argue that if the model believes a swap is likely, the headlines must be part of the same group. The threshold above which $S\left(A_{1}, A_{2}\right)$ predicts a 1 is determined using the training portion of the data. Because this model uses the headline and content of the article, it falls under Challenge 3.

Headline Gen. Swap + Time corresponds to the Headline Generator Swap model, adding publication date information similarly to the Electra MRPC Zero-shot + Time model:

$$
S^{\prime}\left(A_{1}, A_{2}\right)=S\left(A_{1}, A_{2}\right) \cdot e^{-\lambda \Delta T}
$$

This model uses the headline, publication data and content of the article, and falls under Challenge 3.
Unsupervised models were allowed to pick a single hyper-parameter based on training set performance: to learn the threshold in score differentiating between class 1 and class 0 . Strictly speaking, because we tune this single parameter, the methods could be seen as supervised. However, we label them as unsupervised because model parameters were not modified.

\subsection{Supervised Methods}

Electra Finetune stands for an Electra model finetuned on the training set of HLGD, inputting the two headlines, divided by a separator token. Headline order is chosen randomly at each epoch. Because we train a model for several epochs (see Appendix B), a model is likely to see pairs in both orders. This model only uses headlines of articles for prediction, and falls under Challenge 1 .

Electra Finetune on content represents a similar model to that described above, with the difference that the model makes predictions based on the first 255 words of the contents of the two news articles, instead of the headline. This evaluates the informativeness of contents in determining headline groups. This experiment requires the contents and falls under Challenge 3.

Electra Finetune + Time corresponds to an Electra model with time information. The model's output goes through a $768 \times 1$ feed-forward layer, and is concatenated with the day difference of publication, which is run through a $2 \times 2$ feed-forward, and a softmax layer. This model uses headline and time information, and falls under Challenge 2 . 


\subsection{Discussion of Results}

Human performance can be high, close to 0.9 F-1 both on development and test timelines.

Using time alone gives a lower-bound baseline on HLGD, achieving an F-1 of 0.585 on the test set, and confirming that publication date of an article is not enough to perform competitively on HLGD.

Regarding Unsupervised and Zero-shot approaches, the Headliner Generator Swap outperforms Electra MRPC Zero-shot. With additional time information $(+$ time), the generator-based model is able to get close to strong supervised models. The model benefits from pre-training on a large corpus of (content, headline) pairs, having learned a good representation for headlines.

Unsurprisingly, best performance on HLGD is achieved by a supervised approach, Electra Finetune HLGD + Time, which uses both headline and time information. With an F-1 performance on the development set of 0.753 , the model is still 0.13 F-1 points below human performance $(0.07 \mathrm{~F}-1$ difference on the test set).

When finetuning the Electra model with contents instead of headlines, performance drops by $0.07 \mathrm{~F}-1$ points. This is particularly surprising as it could be expected that content contains strictly more information than the headline. We interpret this performance of the content-based model as evidence that the contents are more broad and do not solely focus on the distinguishing fact that is necessary to perform the grouping.

Finally, publication date yields a performance gain of 0.025 to $0.1 \mathrm{~F}-1$ points over models without time information. This confirms that even though time information alone does not achieve high performance, it can be used to enhance models effectively. Because human annotators read timelines chronologically and had access to publication date while annotating, we do not have an upper-bound of human performance without using time.

\section{Analysis of Model Consistency}

Checking whether deep learning models are consistent across predictions has recently become a subject of interest, for example with QA systems with text (Ribeiro et al., 2019) and image (Shah et al., 2019) inputs. We analyze model consistency by probing the Electra Finetune + Time model, which achieves highest performance in terms of F-1 score. We propose a commutative test and transitive test, both illustrated in Figure 5.
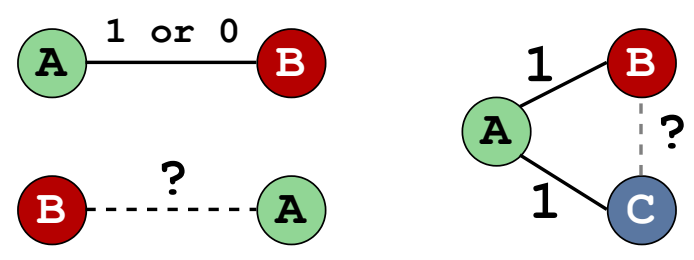

Figure 5: Simplified representation of commutativity (left) and transitivity (right). We verify whether predictions from our best-performing model are consistent with regards to these two properties.

In order to evaluate consistency across training runs, we trained six versions of the Electra Finetune + Time model with the same hyper-parameters. Because each training run processes through the data in a different order, the models are distinct from each other. With regard to performance, the models perform very similarly, achieving within $0.01 \mathrm{~F}-1$ of each other on the development and test sets.

\subsection{Commutative Test}

The HeadLine Grouping task requires two sentences to be compared, both playing a symmetric role.

Most model architectures process the headline pair as a single sequence, and an arbitrary ordering of the pair is chosen for processing. We study whether this arbitrary choice has an impact on the model's prediction. Specifically, we make predictions for all pairs of headlines in the development portion of HLGD, running each pair in both $(A, B)$ and $(B, A)$ order.

On average across the 6 model checkpoints, swapping the order of headlines is enough to make the model change its prediction (put higher probability on 0 in one case and 1 in the other) on $6.3 \%( \pm 0.5)$ of the pairs.

Furthermore, in other cases when the prediction does not change, the probability of the predicted class fluctuates by $0.061( \pm 0.005)$ on average, showing the impact sentence order has on all predictions.

The relatively small standard deviations across training runs indicates that this phenomenon is inherent to the training procedure and not only existent in a subset of models.

A remedy is to build a symmetric classifier:

$$
P_{S}(Y \mid A, B)=\frac{P(Y \mid A, B)+P(Y \mid B, A)}{2}
$$


where $P_{S}$ follows the symmetric rule by design, by predicting for both $\left(H_{1}, H_{2}\right)$ and $\left(H_{2}, H_{1}\right)$ and averaging. When applying this patch to models presented in Section 4, we observe an average gain in F-1 performance of 0.01. Even though encouraging, this gain is a post hoc fix, and enforcing symmetry during training might yield further gains.

\subsection{Transitive Test}

Transitivity involves triplets of headlines A, B and $\mathrm{C}$. The assumption is that if $\mathrm{A}$ and $\mathrm{B}$ are part of the same group, and $\mathrm{A}$ and $\mathrm{C}$ are part of the same group, then B and C must be in the same group as well. The procedure followed during annotation — assigning group IDs to headlines - implies that the transitivity is preserved, as all headline pairs within the same group are positive pairs.

To test a model's consistency with regards to the transitive rule, we use the Electra Finetune + Time model to produce a prediction for all pairs of headlines in the development portion of HLGD.

For each triplet $(\mathrm{A}, \mathrm{B}, \mathrm{C})$ of headlines in the timeline, the model produces three predictions for the $(A, B),(A, C)$, and $(B, C)$ pairs. We focus our attention on triplets where the model has predicted at least 2 positive pairs: if the third pair is predicted to be positive, transitivity is conserved (111 triangle), but if it is predicted to be negative, the triplet breaks the transitivity rule (110 triangle).

On average across the six model checkpoints, we find that of the 60,660 triplets for which the model predicted at least 2 positives pairs, 44,627 triplets had a negative third prediction, and 16,033 had a positive one. In short, the model is consistent only $26.4 \%( \pm 1.4)$ of the time on these triplets.

Improving model consistency with regards to transitivity is challenging, as it would involve presenting the model with triples in some way. Imposing this constraint could yield performance improvements on the task.

We note however that transitivity is a strong assumption, as it is possible for groups of headlines to have stronger and weaker subgroups. It is possible that human annotations would not always follow transitivity if tasked to do so. For this reason, we do not expect models to be $100 \%$ consistent, but there is room for improvement.

\section{Conclusion}

In this work we present the new task of HeadLine Grouping (HLG) a new challenging NLU task, with an accompanying dataset (HLGD). Even though state-of-the-art NLU models have achieved close to human performance on many NLU tasks, we show that there is a considerable gap between best model performance $(0.75 \mathrm{~F}-1)$ and human performance (about $0.9 \mathrm{~F}-1$ ) on HLGD. We therefore propose this dataset as a challenge for future NLU benchmarks. We propose to repurpose a Headline Generator for the task of headline grouping, based on prompting it for the likelihood of a headline swap, and achieve within 3 F-1 of the best supervised model, paving the way for other unsupervised methods to repurpose generators for NLU. Analysis of models on HLGD reveals that they are not consistent in trivial ways, suggesting further improvements needed to NLU models.

\section{Acknowledgments}

We would like to thank the Upwork crowd-workers for their assistance in creating HLGD, as well as Katie Stasaski, Dongyeop Kang and the ACL reviewers for their helpful comments. This work was supported by a Bloomberg Data Science grant. We also gratefully acknowledge support received from an Amazon Web Services Machine Learning Research Award and an NVIDIA Corporation GPU grant.

\section{References}

James Allan. 2002. Introduction to topic detection and tracking. In Topic detection and tracking, pages 116. Springer.

Joshua Bambrick, Minjie Xu, Andy Almonte, Igor Malioutov, Guim Perarnau, Vittorio Selo, and Iat Chong Chan. 2020. Nstm: Real-time querydriven news overview composition at bloomberg. In ACL (System Demonstrations), pages 350-361.

Luisa Bentivogli, Peter Clark, Ido Dagan, and Danilo Giampiccolo. 2009. The sixth pascal recognizing textual entailment challenge. In Text Analysis Conference. NIST.

Vincent D Blondel, Jean-Loup Guillaume, Renaud Lambiotte, and Etienne Lefebvre. 2008. Fast unfolding of communities in large networks. Journal of statistical mechanics: theory and experiment, 2008(10):P10008.

Alireza Bonyadi and Moses Samuel. 2013. Headlines in newspaper editorials: A contrastive study. Sage Open, 3(2):2158244013494863.

Laura Ana Maria Bostan, Evgeny Kim, and Roman Klinger. 2020. GoodNewsEveryone: A corpus of 
news headlines annotated with emotions, semantic roles, and reader perception. In $L R E C$, pages 1554 1566.

Houda Bouamor, Aurélien Max, and Anne Vilnat 2012. Une étude en $3 d$ de la paraphrase: types de corpus, langues et techniques (a study of paraphrase along 3 dimensions: Corpus types, languages and techniques)[in french]. In JEP-TALN-RECITAL 2012, volume 2: TALN, pages 267-280.

Peter Bourgonje, J. M. Schneider, and Georg Rehm. 2017. From clickbait to fake news detection: An approach based on detecting the stance of headlines to articles. In Proceedings of the 2017 EMNLP Workshop: Natural Language Processing meets Journalism, pages 84-89.

Daniel Cer, Mona Diab, Eneko Agirre, Iñigo LopezGazpio, and Lucia Specia. 2017. SemEval-2017 task 1: Semantic textual similarity multilingual and crosslingual focused evaluation. In Proceedings of the 11th International Workshop on Semantic Evaluation (SemEval-2017). Association for Computational Linguistics.

Sudatta Chowdhury and Monica Landoni. 2006. News aggregator services: user expectations and experience. Online Information Review, 30(2):100-115.

Kevin Clark, Minh-Thang Luong, Quoc V. Le, and Christopher D. Manning. 2020. Electra: Pretraining text encoders as discriminators rather than generators. In International Conference on Learning Representations.

Jacob Devlin, Ming-Wei Chang, Kenton Lee, and Kristina Toutanova. 2019. Bert: Pre-training of deep bidirectional transformers for language understanding. In NAACL-HLT), pages 4171-4186.

Bill Dolan, Chris Quirk, and Chris Brockett. 2004. Unsupervised construction of large paraphrase corpora: Exploiting massively parallel news sources. In $C O L$ $I N G$, pages $350-356$.

Seth Flaxman, Sharad Goel, and Justin M Rao. 2016 Filter bubbles, echo chambers, and online news consumption. Public opinion quarterly, 80(S1):298320 .

Rama Rohit Reddy Gangula, Suma Reddy Duggenpudi, and R. Mamidi. 2019. Detecting political bias in news articles using headline attention. In $A C L$ 2019.

David Graff, Junbo Kong, Kazuaki Maeda, and Stephanie Strassel. 2006. Tdt5 multilingual text. Technical Report LDC2006T18, Linguistic Data Consortium, Philadelphia, PA.

Di Jin, Zhijing Jin, Joey Tianyi Zhou, Lisa Orii, and Peter Szolovits. 2020. Hooks in the headline: Learning to generate headlines with controlled styles. In $A C L$, pages 5082-5093.
Aditya Joshi, Kashyap Popat, Shubham Gautam, and P. Bhattacharyya. 2013. Making headlines in hindi: Automatic English to Hindi news headline translation. In IJCNLP.

Philippe Laban and Marti A Hearst. 2017. newsLens: building and visualizing long-ranging news stories. In Proceedings of the Events and Stories in the News Workshop (ACL 2017), pages 1-9.

Vladimir I. Levenshtein. 1966. Binary codes capable of correcting deletions, insertions and reversals. Soviet physics. Doklady, 10:707-710.

Andrew Moore and Paul Rayson. 2017. Lancaster a at SemEval-2017 task 5: Evaluation metrics matter: predicting sentiment from financial news headlines. In Proceedings of the 11th International Workshop on Semantic Evaluation (SemEval-2017), pages 581-585, Vancouver, Canada. Association for Computational Linguistics.

Asli Eyecioglu Ozmutlu. 2016. Paraphrase identification using knowledge-lean techniques. Ph.D. thesis, University of Sussex.

Ekaterina Pronoza, Elena Yagunova, and Anton Pronoza. 2015. Construction of a Russian paraphrase corpus: unsupervised paraphrase extraction. In Russian Summer School in Information Retrieval, pages 146-157. Springer.

Alec Radford, Jeffrey Wu, Rewon Child, David Luan, Dario Amodei, and Ilya Sutskever. 2019. Language models are unsupervised multitask learners. OpenAI blog, 1(8):9.

Pranav Rajpurkar, Robin Jia, and Percy Liang. 2018. Know what you don't know: Unanswerable questions for SQuAD. In ACL (Short Papers), pages 784-789.

Marco Tulio Ribeiro, Carlos Guestrin, and Sameer Singh. 2019. Are red roses red? evaluating consistency of question-answering models. In $A C L$, pages 6174-6184.

Alexander M. Rush, Sumit Chopra, and Jason Weston. 2015. A neural attention model for abstractive sentence summarization. In EMNLP, pages 379-389.

Meet Shah, Xinlei Chen, Marcus Rohrbach, and Devi Parikh. 2019. Cycle-consistency for robust visual question answering. In Proceedings of the IEEE Conference on Computer Vision and Pattern Recognition, pages 6649-6658.

Yusuke Shinyama, Satoshi Sekine, and Kiyoshi Sudo. 2002. Automatic paraphrase acquisition from news articles. In Proceedings of the second international conference on Human Language Technology Research, pages 313-318. Morgan Kaufmann Publishers Inc. 
Ashish Vaswani, Noam Shazeer, Niki Parmar, Jakob Uszkoreit, Llion Jones, Aidan N Gomez, Łukasz Kaiser, and Illia Polosukhin. 2017. Attention is all you need. In Advances in neural information processing systems, pages 5998-6008.

Nguyen Xuan Vinh, Julien Epps, and James Bailey. 2010. Information theoretic measures for clusterings comparison: Variants, properties, normalization and correction for chance. The Journal of Machine Learning Research, 11:2837-2854.

Adina Williams, Nikita Nangia, and Samuel Bowman. 2018. A broad-coverage challenge corpus for sentence understanding through inference. In $N A A C L$, pages $1112-1122$.

Sander Wubben, Antal Van Den Bosch, Emiel Krahmer, and Erwin Marsi. 2009. Clustering and matching headlines for automatic paraphrase acquisition. In Proceedings of the 12th European Workshop on Natural Language Generation (ENLG 2009), pages 122-125.

W. Xu, Alan Ritter, Chris Callison-Burch, W. Dolan, and Yangfeng Ji. 2014. Extracting lexically divergent paraphrases from twitter. Transactions of the Association for Computational Linguistics, 2:435448.

\section{A Annotator Instructions}

The wording of the prompt given to the eight crowd annotators we recruited was the following:

Your task will be to annotate News Headline timelines, and decide which are referring to the same event.

You are given a list of news headlines in chronological order, with a headline on each line of a Spreadsheet. For each headline, the task is to assign it a number: either a new number if the headline represents a new event that hasn't appeared yet, or the number of the existing headline it is a "repetition" of.

For each headline, you are also given a date of publication, which you can use to determine whether two headlines should be in the same event as well: two headlines several months apart must be about different events, even if they are very close lexically (protests in June and September are different events).

In some cases, headlines in the timelines were too vague or did not describe an event specifically, and annotators were encouraged to put such headlines in a group of their own.

\section{B Model Size and Hyper-parameters}

In order to ease reproducibility, we report relevant hyper-parameters of models whose results are present in Section 4. We used implementation of Transformer models from the HuggingFace Transformer library ${ }^{5}$. For Electra models, we initialized using the electra-base-discriminator. For GPT-2 based models, we initialized with the gpt 2 model (which corresponds to a base model as well). Additional model-specific details:

- Electra MRPC Zero-shot: The model produces a probability for label $1: P(Y=1 \mid X)$. If this probability is above a threshold $T$, the model predicts a 1 , and below it predicts a 0 . $T=0.23$ for this model.

- Electra MRPC Zero-shot + Time: We use a time constant of $\lambda=0.15$, and $T=0.14$ for this model.

- Headline Generator Swap: the threshold for this model is $T=0.0012$. This might seem small, but it is the conditional probability of a headline according to the GPT -2 model, and corresponds to a log-probability of -6.75 .

- Headline Gen. Swap + Time: we use a time constant $\lambda=0.07$, and a threshold of $T=0.00056$, which corresponds to a logprobability of -7.49 .

- Supervised models: All supervised models are trained for 3 epochs, with a batch size of 32, and the Adam Optimizer with a learning rate of $L R=10^{-5}$ with an exponential decay and a linear-warmup over the first 1000 steps. All weights of the model are finetuned.

\section{HLGD Format and Removal process}

The dataset is a JSON file that can be processed using standard JSON parsing libraries. Each entry in the JSON object follows the schema:

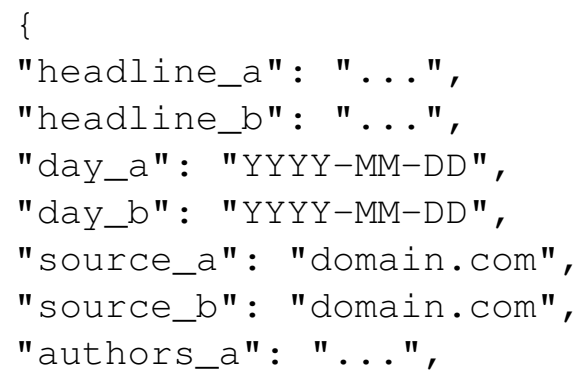

${ }^{5}$ https://github.com/huggingface/transformers 


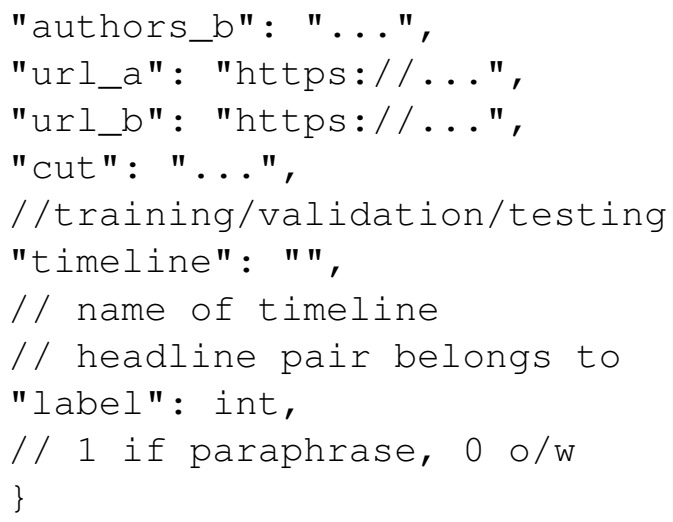

The dataset will include scripts for processing the url for accessing the full content of the articles and other article data and for an option for content owners to request removal.

\section{Excerpt of Timeline}




\begin{tabular}{|c|c|c|c|}
\hline $\begin{array}{l}\text { Publication } \\
\text { Date }\end{array}$ & Source & Headline & Group \\
\hline 2015-01-14 & $\mathrm{cnn}$ & Astronauts relocate after false alarm & 1 \\
\hline 2015-01-14 & bloomberg & Space Station Crew Returns After Alarm Scare Prompts Evacuation & 1 \\
\hline 2015-01-14 & bloomberg & Space Station Crew Safe After Coolant-Pressure Alarm Sounds (1) & 1 \\
\hline 2015-01-14 & foxnews & 6 evacuate US part of space station; NASA says all are safe & 1 \\
\hline 2015-01-15 & reuters & Astronauts back in U.S. part of space station after leak scare & 1 \\
\hline 2015-01-15 & reuters & Crew evacuates U.S. section of space station after leak-agencies & 1 \\
\hline 2015-01-15 & nytimes & $\begin{array}{l}\text { Space Station Crew Temporarily Moves to Russian Side Over Fears of } \\
\text { Ammonia Leak }\end{array}$ & 1 \\
\hline 2015-01-17 & washingtonpost & A false alarm for crew on the International Space Station & 1 \\
\hline 2015-08-10 & telegraph & Astronauts declare first space salad 'awesome' & 2 \\
\hline 2015-08-10 & cnn & Space-grown vegetables: Astronauts chow down on lettuce & 2 \\
\hline 2015-08-10 & foxnews & $\begin{array}{l}\text { For the First Time Ever, NASA Astronauts Eat Vegetables Grown in } \\
\text { Space }\end{array}$ & 2 \\
\hline 2015-08-10 & foxnews & Space Station astronauts make history, eat first space-grown veggies & 2 \\
\hline 2015-08-10 & businessinsider & First space-grown lettuce on the menu today for NASA astronauts & 2 \\
\hline 2015-08-11 & nytimes & Growing Vegetables in Space, NASA Astronauts Tweet Their Lunch & 2 \\
\hline 2016-11-16 & ap & NASA astronaut on verge of becoming oldest woman in space & 3 \\
\hline 2016-11-16 & washingtonpost & Astronaut to become oldest woman to travel in space & 3 \\
\hline 2016-11-17 & france24 & Haute cuisine: top French chefs' food bound for space station & 4 \\
\hline 2016-11-17 & ap & Rocket carrying crew of 3 blasts off for Int'l Space Station & 5 \\
\hline 2016-11-17 & reuters & Multinational crew blasts off, bound for space station & 5 \\
\hline 2016-11-17 & $\mathrm{rt}$ & New ISS crew sets off into space from Russian launchpad (LIVE) & 5 \\
\hline 2016-11-17 & france 24 & Three astronauts blast off to ISS & 5 \\
\hline 2016-11-17 & foxnews & Rocket carrying crew of 3 blasts off for International Space Station & 5 \\
\hline 2016-11-17 & $\mathrm{bbc}$ & Peggy Whitson: Oldest woman in space blasts off to ISS & 5 \\
\hline 2016-11-18 & telegraph & $\begin{array}{l}\text { Nasa veteran Peggy Whitson becomes the oldest woman in space as she } \\
\text { arrives at the ISS }\end{array}$ & 5 \\
\hline 2016-11-18 & theguardian & Oldest woman in space blasts off again for third ISS mission & 5 \\
\hline 2016-11-18 & bbc & Peggy Whitson: Blast off to the ISS for oldest woman in space & 5 \\
\hline 2016-11-19 & telegraph & Russian spaceship delivers three astronauts to space station & 5 \\
\hline 2016-11-19 & ap & Space station receives oldest female astronaut, bit of Mars & 5 \\
\hline 2016-11-20 & foxnews & Space station welcomes the oldest woman astronaut, and a bit of Mars & 5 \\
\hline 2016-11-20 & france 24 & Space station welcomes Frenchman and world's oldest astronaut & 5 \\
\hline 2016-11-23 & bbc & Waste not, want not & 6 \\
\hline 2016-11-24 & france24 & French astronaut Pesquet describes first days aboard space station & 7 \\
\hline 2016-11-25 & telegraph & $\begin{array}{l}\text { French astronaut lands on International Space Station - and is asked to } \\
\text { fix the loo }\end{array}$ & 7 \\
\hline 2017-05-12 & bloomberg & NASA Rejects Idea of Humans on First Flight of New Rocket & 8 \\
\hline 2017-05-12 & bloomberg & NASA Study Warns Against Putting Crew On Huge Rocket's First Flight & 8 \\
\hline 2017-05-13 & reuters & NASA delays debut launch of $\$ 23$ billion moon rocket and capsule & 9 \\
\hline 2018-10-03 & france 24 & NASA skeptical on sabotage theory after mystery ISS leak & 10 \\
\hline 2018-10-03 & theguardian & Nasa casts doubt on Russian theory ISS air leak was sabotage & 10 \\
\hline 2018-10-03 & independent & $\begin{array}{l}\text { Nasa casts doubt on claims International Space Station leak was deliber- } \\
\text { ate }\end{array}$ & 10 \\
\hline 2018-10-04 & france 24 & Astronauts return to Earth from ISS amid US-Russia tensions & 11 \\
\hline 2018-10-04 & france 24 & ISS astronauts return to Earth amid US-Russia tensions & 11 \\
\hline 2019-04-11 & nytimes & Scott Kelly Spent a Year in Orbit. His Body Is Not Quite the Same. & 12 \\
\hline 2019-04-11 & $\mathrm{cnn}$ & $\begin{array}{l}\text { Human health can be 'mostly sustained' for a year in space, NASA Twins } \\
\text { Study concludes }\end{array}$ & 12 \\
\hline 2019-04-11 & bloomberg & NASA's Twins Study Sees No Red Flags for Human Space Travel & 12 \\
\hline 2019-04-11 & independent & Space is doing strange things to astronauts' bodies, Nasa study reveals & 12 \\
\hline 2019-04-11 & reuters & $\begin{array}{l}\text { Oh, brother! NASA twins study shows how space changes the human } \\
\text { body }\end{array}$ & 12 \\
\hline 2019-04-11 & france 24 & NASA's 'Twins Study,' landmark research for an eventual Mars mission & 12 \\
\hline
\end{tabular}

Table A1: Excerpt of the timeline about the International Space Station in HLGD. Group is the global group, aggregating labels from the five annotators. The full timeline contains 257 headlines and 107 distinct groups. 